\title{
ON IDENTIFYING HYPERBOLIC 3-MANIFOLDS AS LINK COMPLEMENTS IN THE 3-SPHERE
}

\author{
DUBRAVKO IVANŠIĆ \\ Murray State University, USA
}

\begin{abstract}
We give a straightforward method that helps recognize when a noncompact hyperbolic 3-manifold is a link complement in the 3sphere and automatically produces the link diagram. The method is based on converting a side-pairing to a handle decomposition.
\end{abstract}

\section{INTRODUCTION}

A number of papers since the '70's have shown that some hyperbolic 3manifolds are link complements in $S^{3}$ (see [CR98] for a survey of results about this topic). However, proofs that a particular manifold is a complement of a particular link typically go in the opposite direction, that is, they construct a hyperbolic structure on a link complement, rather than start with a given manifold and show that it is a link complement in $S^{3}$. In this note we give a method that starts with a noncompact hyperbolic 3-manifold - given by a side-pairing of a polyhedron - and attempts to show that the manifold is a link complement $S^{3}$. If successful, it automatically produces the link diagram.

For context, we briefly recall some of the proofs from the literature that show a homeomorphism between a link complement and a hyperbolic 3-manifold. In the well-known approaches of Thurston's lecture notes ([Thu82, Thu97]), the Whitehead and Borromean links and the figure-eight knot diagrams are the starting points of a cellular decomposition which is brought into correspondence with a side-pairing on hyperbolic polyhedra. A similar approach is taken by Weeks' program SnapPea ([Wee]), which, given the diagram of a link, finds (if possible) an ideal hyperbolic triangulation for the link-complement.

2010 Mathematics Subject Classification. 57M50, 57M25.

Key words and phrases. Handle decomposition, hyperbolic manifold, link complement. 
Another approach is based on Riley's work that investigated representations of link groups in $P S L(2, F)$, where $F$ is a field. In [Ril75b], he found a discrete representation of the figure-eight knot group in $P S L(2, \mathbf{C})$, the first example of a hyperbolic knot complement. The following theorem ([Ril75a]) was the basis for more examples: for a hyperbolic manifold $M$ and a link $L$, if $\pi_{1} M$ is anti-isomorphic to $\pi_{1}\left(S^{3}-L\right)$, then $M \cong S^{3}-L$. Riley ([Ril82]) and Wielenberg ([Wie78]) used it to generate further examples of hyperbolic link-complements by finding an anti-isomorphism between the presentations of groups $\pi_{1} M$ (coming from a side-pairing) and $\pi_{1}\left(S^{3}-L\right)$ (coming from a Wirtinger presentation of the link group). This approach again requires knowing the link first, and computer assistance or a fair amount of trial-anderror (we surmise) made them work.

A computer-assisted investigation of which hyperbolic 3-manifolds are link complements was carried out by Callahan, Dean and Weeks in [CDW99]. The search is conducted for manifolds arising from side-pairings of up to 6 ideal tetrahedra, and proceeds in an indirect fashion: a candidate manifold's volume is compared to the volumes of known hyperbolic knots, and, when they are equal, the manifold is checked to be isometric to the link complement using SnapPea, thus relying on knowing the knot first. Two candidate manifolds resisted this approach and they had to be dealt with separately using special insight and SnapPea.

We are aware of only one proof that does not require knowing the link in advance. It is for the figure-eight knot complement, described in Francis' book [Fra87], p. 153-155, but appears to be significantly restricted by the type of the side-pairing (that proof is in turn inspired by an example of a hyperbolic manifold with genus- 2 surface boundary from the 1982 edition of Thurston's notes [Thu82], also found in [Thu97], Example 3.3.12.).

Our method uses standard dualization of cells to convert the polyhedron and its side-pairing into a handle decomposition of the manifold, as described in $\S 2$. Next, Dehn filling is performed on the torus boundary components by adding a 2-handle. Using handle moves, the handle-decomposition diagram is simplified. If we arrive at the diagram of a 3 -sphere (for example, no 1-handles), we have shown that the manifold is a link complement in $S^{3}$. Tracking the longitudes of the added solid tori yields the link diagram. We illustrate this process on an example of Wielenberg's in $\S 3$.

This method works in a straightforward way on all the standard examples (complements of the figure- 8 knot, the Whitehead link and the Borromean rings) and some less standard ones, like those in [Wie78], the 3-dimensional examples of [RT00], and the two manifolds requiring special treatment in [CDW99]. We trust it will work on many others.

The reader may be interested to know that some hyperbolic 4-manifolds are "link" complements in a topological $S^{4}$, see [Iva04, IRT05] for twelve such examples (in contrast with dimension 3 , however, only finitely many examples 

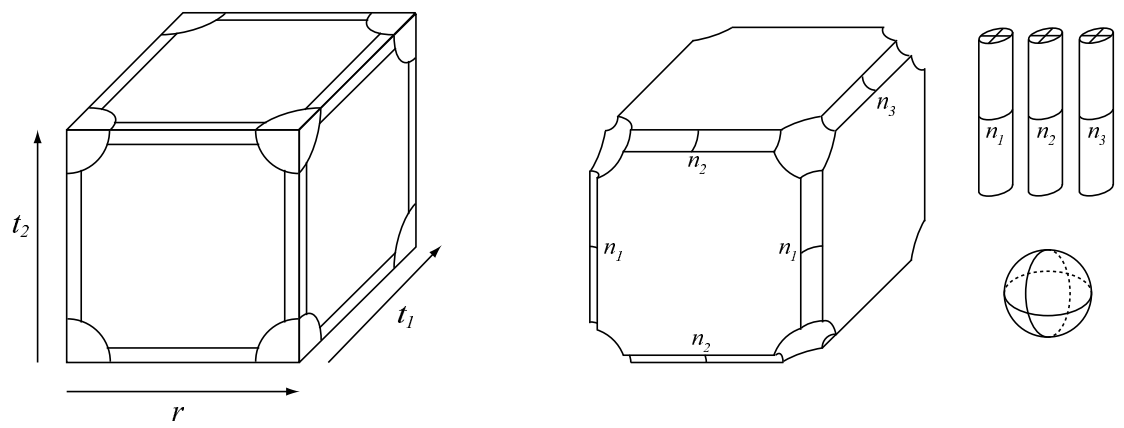

FiguRE 1. Left, cube with side-pairing and neighborhoods of faces, right, handles as assemblies of face neighborhoods

are possible ([Iva04])). An interesting question is whether those topological 4spheres are diffeomorphic to the standard 4-sphere, answered in the affirmative for one example by the author in [Iva] using the same method of converting a polyhedron with a side-pairing to a handle decomposition. As a matter of fact, this was the author's first application of the conversion method, and the one presented in this paper was found afterwards.

\section{Converting A SIDE-PAIRING TO A HANDLE DECOMPOSITION}

Let $P$ be a polyhedron in $X=\mathbf{H}^{3}, \mathbf{E}^{3}$ or $\mathbf{S}^{3}$ with a side-pairing defined on it that gives a geometric manifold $M$. On the left in Fig. 1, a cube is drawn as an example: its top and bottom and front and back sides are paired by a translation, while the left and right sides are paired by a translation followed by a $180^{\circ}$ rotation around the translation vector.

Select neighborhoods (for example, $\epsilon$-neighborhoods) around vertices and edges like in Fig. 1. The neighborhoods should match via the side pairing (see [Iva] for a precise formulation). Let $V_{1}, \ldots, V_{m}$ be neighborhoods of a cycle of vertices $\left\{v_{1}, \ldots, v_{k}\right\}$ (a cycle of faces comprises all the faces of $P$ that are identified by the side-pairing). Then $V_{1} \cup \cdots \cup V_{m}$ assembles into a ball $V$ in $M$. In our example, all the vertices are in the same cycle, and $V_{i}$ is an eighth of a ball. Eight such pieces, of course, assemble in a ball.

Removing neighborhoods of all vertices from $P$ removes parts of the neighborhoods of the edges. Let $E_{1}, \ldots, E_{n}$ be the truncated neighborhoods of a cycle of edges $e_{1}, \ldots, e_{n}$. Then $E_{1} \cup \cdots \cup E_{n}$ assembles into a solid cylinder around a truncated edge, which can also be viewed as a 3-ball $E$ in $M$.

Let $H_{1}$ be the solid obtained by removing neighborhoods of vertices and truncated neighborhoods of edges from $P$. On the surface of $H_{1}$ it is the truncated sides that get identified, representing pairwise-identified disjoint disks, so $H_{1}$ projects to a handlebody $H$ in $M$ under the quotient map $P \rightarrow M$. The 
feet of the 1-handles of $H$ are the truncated sides on $H_{1}$ (see [GS99] for basics of handles and handle decompositions). Now, the ball $E=D^{2} \times D^{1}$ from above is attached to $H$ along $\partial D^{2} \times D^{1}$, making it a 2-handle of $M$. In our example, there are three cycles of edges, and the visible portions of attaching circles $n_{1}, n_{2}$ and $n_{3}$ of the corresponding 2-handles are shown on the right of Fig. 1. Of course, the ball $V$ from above may be viewed as $V=D^{3} \times D^{0}$, and it attaches to the 0-, 1- and 2-handles along $\partial D^{3} \times D^{0}$, making it a 3-handle.

If $P$ is a polyhedron in $\mathbf{H}^{3}$ with some ideal vertices, the procedure works the same way, except, instead of removing a neighborhood of the vertex we remove a horoball centered at the ideal vertex.

Therefore, to get a handle decomposition diagram (pairs of disks in $\mathbf{R}^{2}$ representing feet of 1-handles, curves outside of the disks representing attaching circles of 2-handles), do the following:

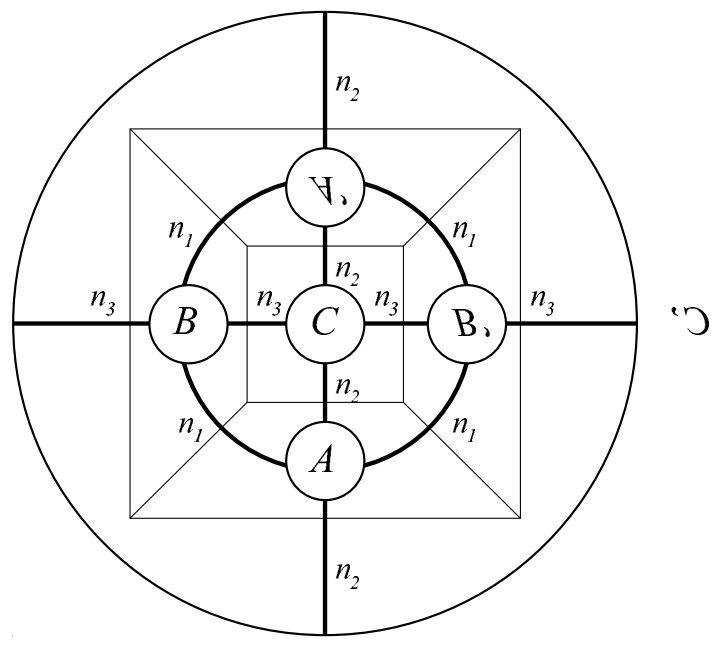

FiguRE 2. Handle decomposition for the side-pairing from Fig. 1

- Project the surface of the polyhedron $P$ to $\mathbf{R}^{2} \cup \infty$ and draw its decomposition into sides (if the polyhedron has ideal vertices, one may draw them as empty circles).

- Draw a disk inside every side that represents one of the feet of a 1handle (paired sides correspond to feet of 1-handles). One of the disks may be the outside of the diagram since a sphere (the surface of $P$ ) was projected to $\mathbf{R}^{2}$.

- If two sides are adjacent along an edge $e$, draw an arc crossing $e$ once between the disks corresponding to the sides. The union of arcs crossing edges that are in the same cycle comprise the attaching circle for a 2-handle. 
- Attention needs to be paid to how disks (feet of 1-handles) are identified, as the transformation that identifies them depends on the transformation that identifies the corresponding sides of $P$ (we do not assume that the feet of 1-handles are identified by a reflection in the bisector of the centers, as is common in handle-decomposition diagrams).

- It is not necessary to keep track of 3-handles, since there is only one way to attach them. Furthermore, if the polyhedron is hyperbolic and has only ideal vertices, there are no 3 -handles. However, if some of the vertices are real and some ideal, it may be useful to note where on the diagram the 3 -handles attach.

Fig. 2 illustrates the process above for the cube example at the beginning of the section. The letters inside the disks suggest the map that pairs the two disks, for example, $A$ and $A^{\prime}$ are paired by a reflection in their bisector, while $B$ and $B^{\prime}$ are paired by a reflection in the bisector, followed by a rotation by $180^{\circ}$.

\section{IDENTIFYING HYPERBOLIC 3-MANIFOLDS AS LINK COMPLEMENTS IN THE 3-SPHERE}

In this section, we apply the conversion method of $\S 2$ to illustrate a procedure that attempts to show that a finite-volume noncompact hyperbolic manifold is the complement of a link in the 3-sphere. If the procedure is carried out successfully, it also produces the link diagram.

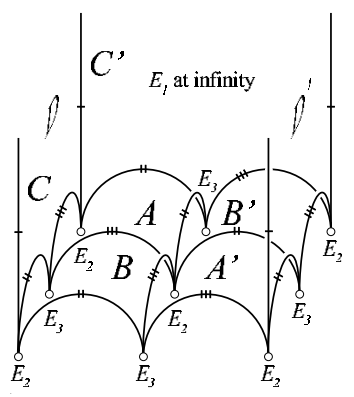

FiguRE 3. Wielenberg's side-pairing on a hyperbolic polyhedron

We will use Wielenberg's example 4 from [Wie78]. This hyperbolic manifold comes from pairing the sides of the polyhedron $P$ pictured in the upper half-space model in Fig. 3. The vertical sides $C, C^{\prime}$ and $D, D^{\prime}$ are paired by translations. Sides $A$ and $A^{\prime}$ are paired by a reflection in the vertical plane passing through the point where $A$ and $A^{\prime}$ touch. Side $B$ is sent to $B^{\prime}$ by 


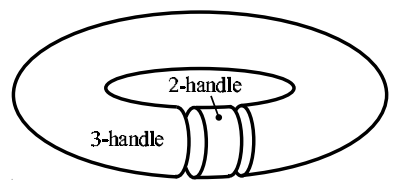

Figure 4. Attaching a solid torus along boundary is like adding a 2-handle and a 3-handle

a reflection in the vertical plane that slices $B$ and $B^{\prime}$ in half, followed by a translation that slides $B$ to $B^{\prime}$.

A finite-volume orientable noncompact hyperbolic 3-manifold $M$ is diffeomorphic to the interior of a compact 3-manifold $\bar{M}$, whose boundary components are all tori. If solid tori are glued onto the boundary components and the result is a 3 -sphere, then $M$ is diffeomorphic to $S^{3}-L$, where $L$ is the collection of the center circles of the solid tori we added.

As Fig. 4 suggests, gluing a solid torus to a component $T^{2}$ of $\partial \bar{M}$ is the same as attaching a 2-handle and a 3 -handle to $\bar{M}$. The attaching circle of the 2-handle can be any nontrivial simple closed curve on $T^{2}$. The components of $\partial \bar{M}$ are assembled from polygons, called vertex links, that are intersections of small enough horospheres centered at ideal vertices with the polyhedron $P$. In our example, the vertex links are $45^{\circ}-45^{\circ}-90^{\circ}$ triangles and squares. The three cycles of ideal vertices, $E_{1}, E_{2}$ and $E_{3}$ are indicated in Fig. 3, and in Fig. 5 the vertex links from each cycle are drawn together and it is shown how they assemble into parallelograms that give rise to toral boundary components of $\bar{M}$.

For every boundary component $T^{2}$ we now choose two curves representing generators of $\pi_{1} T^{2}$. One will serve as the attaching circle of the 2-handle, making it a meridian of the attached solid torus. The other automatically becomes a longitude of the solid torus, thus isotopic to its center circle. In Fig. 5, the attaching circle is the thinner $\operatorname{arc} m_{i}$ and the longitude is the thicker arc $l_{i}, i=1,2,3$.

When choosing the attaching circle, choose a curve in $T^{2}$ that is as short as possible in its Euclidean metric. If the length of the attaching circle is more than $2 \pi$, the $2 \pi$-theorem on hyperbolic Dehn surgery ([BH96]) asserts we will get a hyperbolic manifold. Thus, if $\partial \bar{M}$ has only one component, we will have failed to produce $S^{3}$. If $\partial \bar{M}$ has several components, it is possible that some combination of long and short attaching circles still produces $S^{3}$, but chances are better the greater the number of shorter ones are chosen. In all the examples that we worked out, taking the obvious short choices was successful.

Let $M_{W}$ now denote the manifold resulting from the side-pairing on the polyhedron above. Since there are three cycles of ideal vertices, $\partial \bar{M}_{W}$ will 

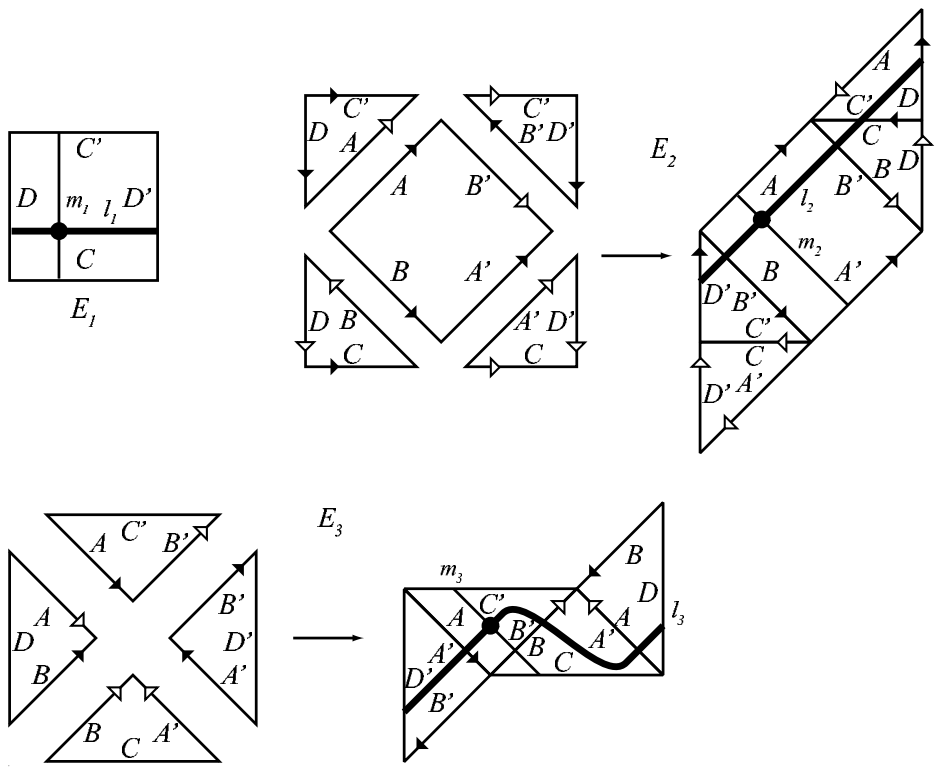

FiguRE 5. Finding suitable meridians in boundary components

have three components. Step 0 of Fig. 7 shows the handle decomposition of $\bar{M}_{W}$, obtained using the conversion method from $\S 2$. The feet $A, A^{\prime}, C, C^{\prime}$ and $D, D^{\prime}$ of 1-handles are all identified by a reflection in the perpendicular bisector of the line connecting their center. The feet $B$ and $B^{\prime}$ are identified by a reflection in the line joining their centers, followed by a translation that moves $B$ to $B^{\prime}$, so that the arrows drawn inside match up. Attaching circles coming from cycles of edges are labeled I, II and III. The attaching circles that we chose in Fig. 5 are also drawn in and labeled $m_{1}, m_{2}$, and $m_{3}$. Their corresponding longitudes $l_{1}, l_{2}$ and $l_{3}$, are drawn as thick curves.

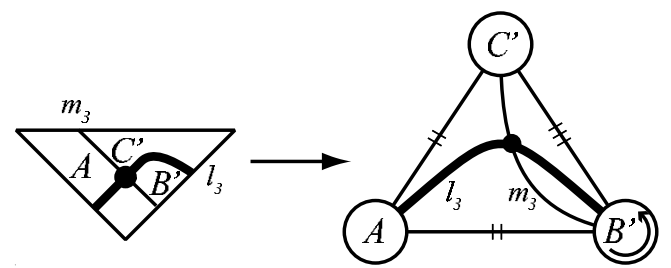

Figure 6. Converting one diagram to another

Fig. 6 shows how to make the easy correspondence between a triangle appearing in Fig. 5 and the section of the boundary of the handlebody in 
step 0 of Fig. 7 necessary to draw in the longitudes and meridians. The handle decomposition of $\bar{M}_{W}$ does not have any 3 -handles, since $P$ did not have any real vertices. However, closing off $\partial \bar{M}_{W}$ with three solid tori adds three 3-handles, which do not need to be tracked in a closed manifold.
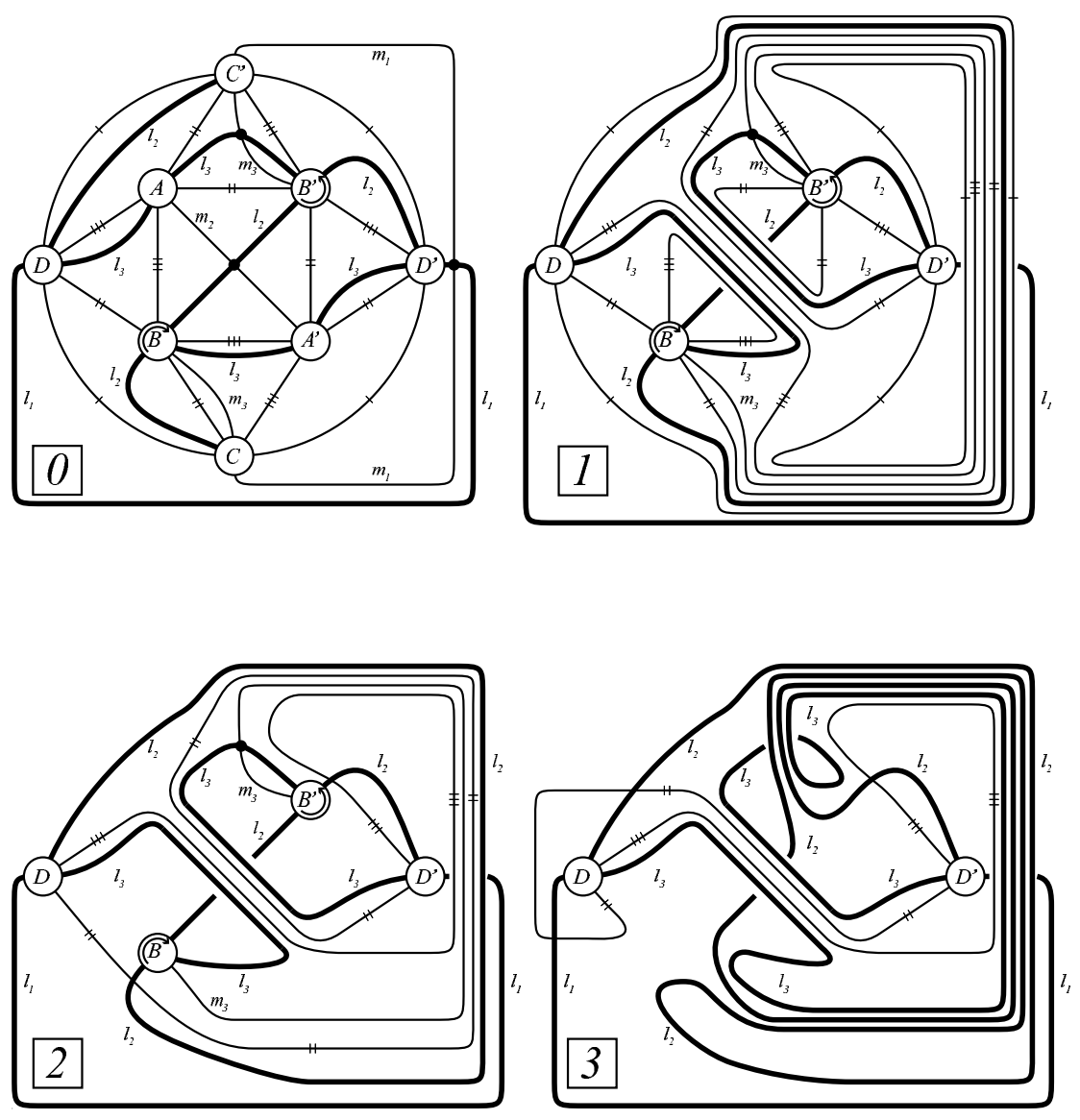

Figure 7. Handle moves, steps $0-3$

Thus, step 0 of Fig. 7 shows the handle decomposition of a closed manifold that we hope is $S^{3}$. In the diagrams in Figures 7 and 8 we perform handle moves in order to simplify the handle decomposition (see [GS99] for basics on handle moves). Keep in mind that the curves labeled $l_{1}, l_{2}$ and $l_{3}$ are not attaching circles, but merely curves drawn on the surface of the handlebody whose position we keep track of. In particular, attaching circles may freely be isotoped over these curves and may cross them. It is easy to see that a crossing 

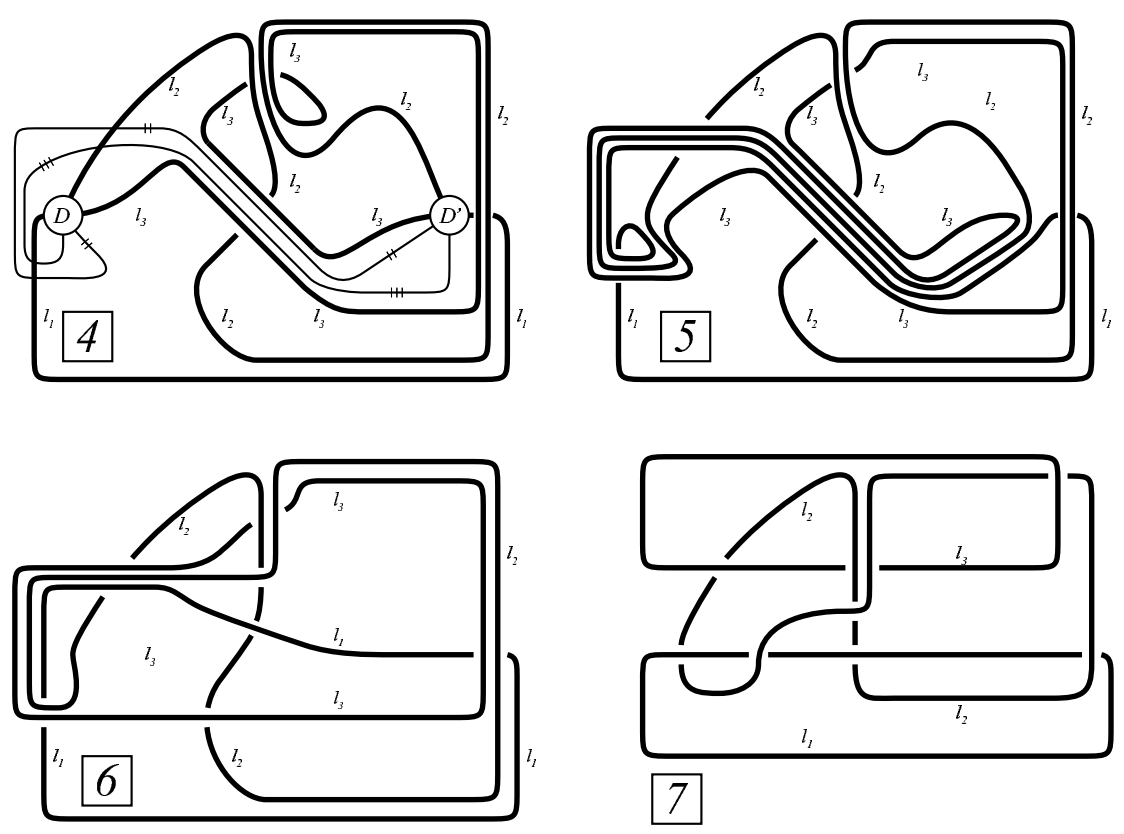

Figure 8. Handle moves, steps $4-7$

by an attaching circle will become an undercrossing if the corresponding 2handle cancels a 1-handle that carries one of the longitudes.

Step 0. Attaching circles $m_{2}$ and $m_{3}$ go across 1-handles $A A^{\prime}$ and $C C^{\prime}$ only once, respectively, so their corresponding 2-handles cancel the 1-handles $A A^{\prime}$ and $C C^{\prime}$. Step 1 shows the handle decomposition after this cancellation.

STEP 1. Attaching circles II and III, which loop from feet $B^{\prime}$ and $B$ can be slid over the 1-handle $B B^{\prime}$ and then off feet $B$ and $B^{\prime}$, respectively. Moreover, the looping part of attaching circle I, at near right, may be isotoped to foot $D^{\prime}$ and then across and off handle $D D^{\prime}$, after which I is a simple closed curve bounding a disk (on the outside) that may be pushed away from the diagram. A 2-handle whose attaching circle bounds a disk disjoint from the rest of the diagram simply encloses a 3-handle if the manifold is compact, like in our case. The 2- and 3-handles then cancel. Step 2 shows the handle decomposition after the isotopies and cancellation.

STEP 2. We now notice that the vertical portion of attaching circle II can be isotoped outside of the diagram to the right and "wrapped" across $\infty$ to its new position shown in Step 3. Attaching circle $m_{3}$ crosses 1-handle $B B^{\prime}$ only once, causing cancellation of the 2-handle corresponding to $m_{3}$ and the 1-handle $B B^{\prime}$. 
STEP 3. Before we carry out further cancellation, we simplify the picture a bit. We isotope attaching circle III around $D^{\prime}$ so it attaches at the bottom. Notice that this includes a slide of the part of III that runs across the 1-handle $D D^{\prime}$, thus the place where III attaches to $D$ moves as well. Also, we isotope the loop of $l_{2}$ at the bottom of the diagram toward the top, and we straighten out the kink in $l_{3}$.

STEP 4. We isotope at top middle to remove the self-crossing of $l_{3}$. Attaching circles II and III run parallel, that is, they bound an annulus. This means a 3 -handle is located between them which cancels one of the 2-handles, say III. After erasing III we note that II cancels the 1-handle $D D^{\prime}$.

STEP 5. The rest is isotopy of the link components $l_{i}, i=1,2,3$. The loop of $l_{3}$ at center right is isotoped up and to the left, and so is the section of $l_{2}$ close to it. The kinks on the left are straightened out, as is the bottom part of $l_{3}$ and the center of $l_{1}$.

STEP 6. The bottom part of $l_{3}$ is lifted and flipped to the top, $l_{1}$ is straightened out and $l_{2}$ is isotoped a little.

STEP 7. After isotoping $l_{2}$ and rotating the diagram by $180^{\circ}$, one gets the mirror image of Fig. 7 from Wielenberg's paper [Wie78].

\section{REFERENCES}

[BH96] S. Bleiler and C. Hodgson, Spherical space forms and Dehn filling, Topology 35 (1996), 809-833.

[CDW99] P. J. Callahan, J. C. Dean and J. R. Weeks, The simplest hyperbolic knots, J. Knot Theory Ramifications 8 (1999), 279-297.

[CR98] P. J. Callahan and A. W. Reid, Hyperbolic structures on knot complements, Chaos Solitons Fractals 9 (1998), 705-738.

[Fra87] G. K. Francis, A topological picturebook, Springer-Verlag, 1987.

[GS99] R. Gompf and A. Stipsicz, 4-manifolds and Kirby calculus, AMS, Providence, 1999.

[IRT05] D. Ivanšić, J. Ratcliffe and S. Tschantz, Complements of tori and Klein bottles in the 4-sphere that have hyperbolic structure, Algebr. Geom. Topol. 5 (2005), 999-1026.

[Iva] D. Ivanšić, A topological 4-sphere that is standard, to appear in Adv. Geom.

[Iva04] D. Ivanšić, Hyperbolic structure on a complement of tori in the 4-sphere, Adv. Geom. 4 (2004), 119-139.

[Ril75a] R. Riley, Discrete parabolic representations of link groups, Mathematika 22 (1975), 141-150.

[Ril75b] R. Riley, A quadratic parabolic group, Math. Proc. Cambridge Philos. Soc. 77 (1975), 281-288.

[Ril82] R. Riley, Seven excellent knots, in: Low-dimensional topology (Bangor, 1979), Cambridge Univ. Press, Cambridge, 1982, 81-151.

[RT00] J. Ratcliffe and S. Tschantz, The volume spectrum of hyperbolic 4-manifolds, Experiment. Math. 9 (2000), 101-125.

[Thu82] W. Thurston, The geometry and topology of three-manifolds, Princeton University lecture notes, 1979, 1982.

[Thu97] W. P. Thurston, Three-dimensional geometry and topology. Vol. 1, Princeton University Press, Princeton, 1997, edited by Silvio Levy. 
[Wee] J. Weeks, Snappea: a computer program for creating and studying hyperbolic 3-manifolds, available at http://www.geometrygames.org/SnapPea/.

[Wie78] N. Wielenberg, The structure of certain subgroups of the Picard group, Math. Proc. Cambridge Philos. Soc. 84 (1978), 427-436.

D. Ivanšić

Department of Mathematics and Statistics

Murray State University

Murray, KY 42071

USA

E-mail: divansic@murraystate.edu

Received: 31.10.2011.

Revised: 5.6.2012. 